\title{
Estudio de la resistencia a compresión de morteros fabricados con conglomerante compuesto de polvo de vidrio
}

\author{
Study of the compressive stregth of mortars made \\ with binders composite glass powder
}

L. Marco $^{(*)}$, E. García(*), M.I. Más(*), V. Alcaraz ${ }^{(*)}$, A. Luizaga ${ }^{(*)}$

RESUMEN

Esta investigación evalúa la resistencia de morteros realizados con mezclas de diferentes composiciones, donde una parte del conglomerante tradicional es sustituido por el polvo de vidrio para analizar de una forma sencilla las características mecánicas de las muestras reactivas obtenidas de esta forma. Los ensayos se han realizado con morteros.

Para facilitar las reacciones se añaden a las mezclas objeto del análisis diferentes reactivos: se mezcla el polvo de vidrio con reactivos básicos, lo que permite la obtención de condiciones de $\mathrm{pH}$ superiores a 12, en las que el vidrio sodo-cálcico es muy reactivo.

Analizadas las diferentes probetas de morteros, se observa que el polvo de vidrio, así como su granulometría, juegan un importante papel en el aumento de las resistencias mecánicas a compresión de las mezclas.

$681-5$

Palabras clave: Mortero; polvo de vidrio; resistencia; conglomerante.

\section{SUMMARY}

This research evaluates the resistance of mortars made with mixtures of different compositions, where a part of the traditional binder is replaced by glass power in a simple way to analyze the mechanical properties of the reactive samples obtained in this way. The tests were carried out with mortars.

To facilitate the reactions are added to the mixtures being analyzed different reagents, mixing the glass powder with basic reagents, allowing the collection of conditions of $\mathrm{pH}$ greater than 12 , ins which the soda-lime glass is very reactive.

Analyzed the different specimens of mortar, shows, that the glass powder and its fineness, play an important role in the increasing the compressive strength of the mixtures.

Keywords: Mortar; glass powder; strench; binder.

(*) EUITOP - Universidad Politécnica de Madrid, (España). 


\section{INTRODUCCIÓN}

Hoy en día, donde la protección al medioambiente se impone como condición necesaria, el reciclaje y la reutilización de materias y recursos, constituyen la base de las políticas de la gestión ambiental en las empresas.

Dentro de este marco se engloba el proceso de reciclado del vidrio. El vidrio es un material que por sus características físicoquímicas es fácilmente reciclable. Este proceso conlleva un gran ahorro en cuanto al gasto de materias primas y energía, lo que supone una minimización de los impactos medioambientales generados en los procesos de fabricación, así como un beneficio económico directo (1) (2).

Tabla 1. Composición del polvo de vidrio empleado en la elaboración del producto

\begin{tabular}{|c|c|c|}
\hline Óxido & Vidrio & Residuo \\
\hline $\mathrm{SiO}_{2}$ & 71,00 & 62,13 \\
\hline $\mathrm{Na}_{2} \mathrm{O}$ & 11,80 & 3,57 \\
\hline $\mathrm{K}_{2} \mathrm{O}$ & 0,60 & 3,49 \\
\hline $\mathrm{CaO}$ & 11,28 & 9,27 \\
\hline $\mathrm{MgO}$ & 1,40 & 0,40 \\
\hline $\mathrm{MnO}$ & 0,10 & 0,00 \\
\hline $\mathrm{Al}_{2} \mathrm{O}_{3}$ & 2,20 & 8,74 \\
\hline $\mathrm{Fe}_{2} \mathrm{O}_{3}$ & 1,60 & 0,24 \\
\hline $\mathrm{TiO}_{2}$ & 0,07 & 0,00 \\
\hline $\mathrm{P}_{2} \mathrm{O}_{5}$ & 0,05 & 0,15 \\
\hline $\mathrm{SO}_{3}$ & 0,00 & 0,00 \\
\hline $\mathrm{Cr}_{2} \mathrm{O}_{3}$ & 0,00 & 0,00 \\
\hline $\mathrm{CO}_{3} \mathrm{O}_{4}$ & 0,00 & 0,00 \\
\hline $\mathrm{ZnO}$ & 0,00 & 5,23 \\
\hline $\mathrm{SrO}$ & 0,00 & 0,10 \\
\hline $\mathrm{ZrO}$ & 0,00 & 0,07 \\
\hline $\mathrm{BaO}$ & 0,00 & 0,00 \\
\hline $\mathrm{PbO}$ & 0,00 & 0,00 \\
\hline $\mathrm{HfO}$ & 0,00 & 0,10 \\
\hline $\mathrm{Rb}{ }_{2} \mathrm{O}$ & 0,00 & 0,10 \\
\hline $\mathrm{CdO}$ & 0,00 & 0,10 \\
\hline $\mathrm{B} 2 \mathrm{O} 3$ & 0,00 & 6,31 \\
\hline $\mathrm{PF} / \mathrm{Volátil}$ & 0,90 & 0,00 \\
\hline $\mathrm{TOTAL}$ & 100,00 & 100,00 \\
\hline & & \\
\hline
\end{tabular}

Residuos: fritas procedentes de la industria cerámica.

Tabla 2. Granulometría del cemento CEM I 52,5 R

\begin{tabular}{|c|c|}
\hline $\begin{array}{c}\text { Diámetro de } \\
\text { las partículas } \\
\text { en }(\mu \mathrm{m})\end{array}$ & $\begin{array}{c}\text { Fracción } \\
\text { volumétrica } \\
\text { en }(\%)\end{array}$ \\
\hline$<8$ & 41,5 \\
\hline$<96$ & 99,7 \\
\hline
\end{tabular}

En el proceso de reciclado se emplean todo tipo de deshechos de vidrio, procedentes de la recuperación selectiva de eny cerámica. Salvo un pequeño porcentaje de éstos, que no pueden ser empleados por su alto contenido en papel, corcho $u$ otras materias, y que son depositados en vertederos controlados constituyen un importante problema medioambiental y económico (1) (2).

Ante esta situación, con este estudio se plantea un sistema de valoración de dichos desechos que podrán ser utilizados en diferentes campos de la ingeniería civil. De esta forma, se genera un doble beneficio, económico y ambiental: por un lado una disminución del volumen de los residuos acumulados en vertederos controlados y por otro la generación de unos importantes ingresos adicionales por su utilización como materia prima en la construcción de obras de ingeniería civil.

El objetivo del estudio es analizar las resistencias a compresión de morteros fabricados con polvo de vidrio, como sustitutivo de parte del conglomerante tradicional, con diferentes granulometrías y distintas proporciones para su uso en la estabilización de suelos (3).

\section{MATERIALES Y MÉTODOS}

Se han empleado tanto desechos procedentes de envases y embalajes, como productos de desecho de la industria cerámica. La composición química de los materiales usados en la elaboración del polvo de vidrio es la que se refleja en la Tabla 1.

Además en la elaboración de los morteros que sirvieron de base para la elaboración de las probetas normalizadas, se emplearon: vases y embalajes de la industria vidriera
- La cal viva "CaO": La cal viva utilizada procede de la fábrica "Cal de Castilla S.A.", en polvo blanco cuya granulometría está comprendida entre 0 y 200 micras.

- La sosa "NaOH" y la potasa "KOH": La sosa y la potasa utilizadas son de la marca PROLABO. Ambas tienen la forma de polvo de color blanco.

- El cemento CEM I 52,5 R: El cemento Portland utilizado es de tipo CEM I y de clase de resistencia 52,5 R. Proviene de la fábrica de Cementos Portland en Chinchón. La letra $\mathrm{R}$ indica una resistencia a corto plazo elevada. Tiene una masa volumétrica de $3,12 \mathrm{~g} / \mathrm{cm}^{3}$, una superficie específica de $4.440 \mathrm{~cm}^{2} / \mathrm{g}$ y un color gris verdoso. Sus características granulométricas aparecen el la Tabla 2.

La caracterización de la resistencia mecánica de morteros fabricados con conglomerante compuesto de polvo de vidrio, se ha realizado a través del ensayo a compresión de tres probetas normalizadas por amasada y unidad de ensayo, obteniéndose su valor estadístico para la cuantificación de ésta (4). En estas probetas se variaron las características del conglomerante de la siguiente forma:

- Naturaleza de los reactivos empleados junto con el polvo de vidrio.

- Cantidad de polvo de vidrio empleado en relación con los demás reactivos que constituyen el conglomerante.

- Granulometría del polvo de vidrio utilizado en las mezclas.

Cabe indicar que las sustituciones del cemento por otros productos (polvo de vidrio, cal viva, potasa y sosa) para la constitución del conglomerante final, son sustituciones de masas que han sido realizadas sin correcciones volumétricas de los áridos (5). Las resistencias a compresión son expresadas con una variabilidad de $\pm 1,5 \mathrm{MPa}$.

\section{RESULTADOS Y DISCUSIÓN}

\subsection{Ensayos realizados con cal, sosa y potasa}

Al comienzo del ensayo, las probetas realizadas (4) se componen de un conglomerante generado a base de polvo de vidrio, cal y sosa o potasa. Se emplean la sosa y la potasa por ser dos reactivos muy básicos, que confieren a la mezcla un medio reactivo con un $\mathrm{pH}$ elevado, lo que sirve para aumentar la reactividad del vidrio. Pero al tratarse de dos productos cuyo coste es muy elevado, no es conveniente usarlos en la elaboración de cantidades importantes de conglomerante, debiéndose prescindir de ellos cuando se vayan a realizar probetas para el uso en distintos campos de la 


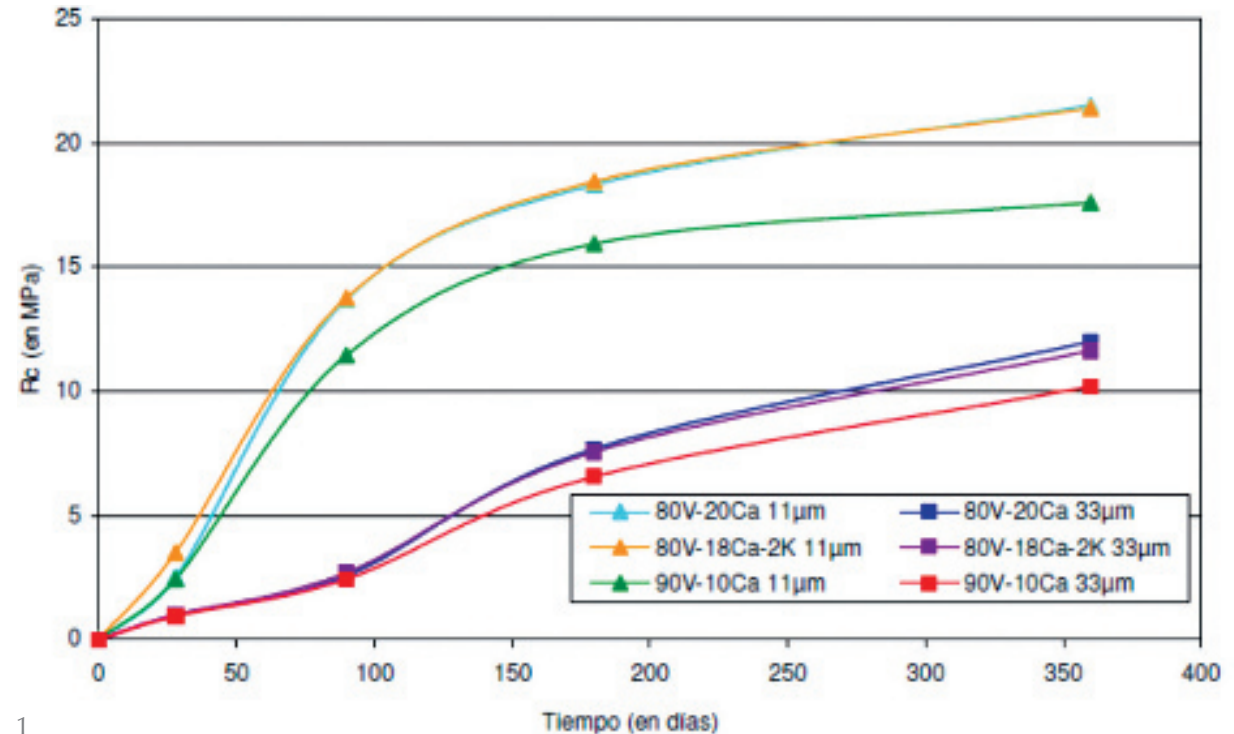

ingeniería civil. En este caso, y a modo de experiencia, estos dos productos sólo formaron parte de nuestras mezclas reactivas en porcentajes del $1 \%$ y del $2 \%$.

Para estudiar la influencia de la granulometría de las partículas constitutivas del polvo de vidrio en las resistencias finales de los morteros elaborados, se preparan éstos con polvo de vidrio, cuyas granulometrías son las siguientes: $d_{50}=33 \mu \mathrm{m}$ y $d_{50}=11 \mu \mathrm{m}$.

La composición final del conglomerante es la siguiente: entre el $80 \%$ y el $100 \%$ de polvo de vidrio, entre el $10 \%$ y el $20 \%$ de cal viva y entre el 1 y el $2 \%$ de sosa o potasa.
1. Evolución de las resistencias a compresión simple de los morteros a base de polvo de vidrio, de cal y de potasa.

Tabla 3. Resultados de las resistencias a la compresión simple de probetas de morteros normalizados en función de las mezclas reactivas utilizadas.

\begin{tabular}{|l|c|c|c|c|c|c|}
\hline $\begin{array}{c}\text { Tipo de } \\
\text { Conglomerante }\end{array}$ & \multicolumn{3}{|c|}{$\begin{array}{c}\text { Conglomerante a base de } \\
\text { polvo de vidrio de } d_{50}=33 \mu \mathrm{m}\end{array}$} & \multicolumn{3}{c|}{$\begin{array}{c}\text { Conglomerante a base de } \\
\text { polvo de vidrio de } \mathrm{d}_{50}=11 \mu \mathrm{m}\end{array}$} \\
\hline $\begin{array}{c}\text { Tipo de mezcla } \\
\text { reactiva }\end{array}$ & $\begin{array}{c}80 \mathrm{~V}-20 \mathrm{Ca} \\
33 \mu \mathrm{m}\end{array}$ & $\begin{array}{c}80 \mathrm{~V}-18 \mathrm{C} a-2 \mathrm{~K} \\
33 \mu \mathrm{m}\end{array}$ & $\begin{array}{c}90 \mathrm{~V}-10 \mathrm{Ca} \\
33 \mu \mathrm{m}\end{array}$ & $\begin{array}{c}80 \mathrm{~V}-20 \mathrm{Ca} \\
11 \mu \mathrm{m}\end{array}$ & $\begin{array}{c}80 \mathrm{~V}-18 \mathrm{C} a-2 \mathrm{~K} \\
11 \mu \mathrm{m}\end{array}$ & $\begin{array}{c}90 \mathrm{~V}-10 \mathrm{Ca} \\
11 \mu \mathrm{m}\end{array}$ \\
\hline$\%$ polvo de vidrio & 80 & 80 & 90 & 80 & 80 & 90 \\
\hline$\%$ cal & 20 & 18 & 10 & 20 & 18 & 10 \\
\hline$\%$ potasa & 0 & 2 & 0 & 0 & 2 & 0 \\
\hline RC (28 días) en MPa & 1,0 & 1,0 & 0,9 & 2,5 & 3,5 & 2,4 \\
\hline RC (90 días) en MPa & 2,6 & 2,7 & 2,4 & 13,7 & 13,8 & 11,4 \\
\hline RC (180 días) en MPa & 7,7 & 7,6 & 6,6 & 18,3 & 18,4 & 15,9 \\
\hline RC (360 días) en MPa & 12,0 & 11,6 & 10,2 & 21,5 & 21,4 & 17,6 \\
\hline
\end{tabular}


En las probetas cuyo conglomerante está constituido por polvo de vidrio únicamente, la resistencia a compresión es nula (6). Se observó también que las probetas que contenían sosa eran equivalentes a las fabricadas con potasa, por lo que en la Figura 1 sólo se han representado las primeras.

Quedó además constatado que el grado de molienda del polvo de vidrio ejercía una importante influencia sobre las resistencias mecánicas obtenidas de los morteros. Cuanto más fino es el polvo de vidrio mayor resistencia se obtiene, independientemente del tiempo transcurrido.

De esta forma, a los 90 días para los morteros formados por polvo de vidrio con $\mathrm{d}_{50}=11 \mu \mathrm{m}$ se obtiene una resistencia a compresión cinco veces mayor que para los fabricados con polvo de vidrio de $\mathrm{d}_{50}=33$ $\mu \mathrm{m}$. Sin embargo, a los 360 días, esta relación es sólo del doble. Por tanto, se puede deducir que las diferencias de los valores de las resistencias a compresión de los morteros fabricados con polvo de vidrio de diferentes granulometrías son muy marcadas en los primeros días, pero estas diferencias tienden a disminuir con el tiempo.

Así mismo, se observa que además de la granulometría del polvo de vidrio, en las variaciones de las resistencias a compresión interviene la cantidad de reactivo utilizado. Los morteros en los que el conglomerante se compone de un porcentaje de cal viva que varía entre el 18 y el $20 \%$, ofrecen resistencias superiores a aquellos en los que el conglomerante contiene sólo un 10\% de este material.

Por el contrario, la adición a la mezcla de potasa parece no jugar un papel demasiado relevante en lo que a la variación de las resistencias a compresión se refiere, ya que sólo se observan crecimientos de resistencias en el periodo de los 0 a los 28 días. Pero este crecimiento mayor, entre los 0 y los 28 días, es muy interesante sobre todo para la aplicación de estas formulaciones en el campo de la estabilización reforzada de suelos, y es siempre preferible la utilización de potasa en lugar de sosa debido a las eflorescencias que pueden formarse con ésta.

La cinética de las reacciones también varía en función de la granulometría del polvo de vidrio empleado en el conglomerante (6).

El incremento de las resistencias a compresión se produce durante un largo periodo de tiempo. Se ha comprobado que entre los 180 y los 360 días se producen aumentos de los valores de las resistencias nada despreciables (entre un 10 y un $50 \%$, de- pendiendo de la granulometría del polvo de vidrio y los reactivos empleados en la elaboración de las probetas).

Para las probetas elaboradas con polvo de vidrio de $\mathrm{d}_{50}=11 \mu \mathrm{m}$, el máximo crecimiento de la resistencia a compresión se sitúa entre los 28 y los 90 días, con un coeficiente multiplicador de 5,5 entre ambas fechas. Para el mismo periodo de tiempo pero para morteros elaborados con polvo de vidrio de granulometría $\mathrm{d}_{50}=33 \mu \mathrm{m}$, el coeficiente multiplicador se reduce a 2,6.

En este último caso, el máximo crecimiento de la resistencia a compresión se produce entre los 90 y los 180 días, con un coeficiente multiplicador en este caso de 3, mientras que para los morteros de polvo de vidrio de granulometrías inferiores este coeficiente para el mismo periodo de tiempo se reduce a 1,3 .

De todo lo anterior se deduce que cuanto más fino es el polvo de vidrio usado en la fabricación de las probetas mayores son las resistencias obtenidas. Por otro lado, el crecimiento de la resistencia a compresión es también mucho más rápido para el polvo fino que para el grueso.

Estos crecimientos de la resistencia a largo plazo no son imputables a las reacciones puzolánicas ordinarias, ya que la cal responsable de este tipo de fraguado debería consumirse bastante rápidamente.

Con el fin de obtener resistencias mayores a largo plazo, se emplea también cemento Portland, el CEM I 52,5 R, puesto que la cal la sosa y la potasa no permiten esperar resistencias mecánicas suficientes para los usos previstos. El cemento empleado juega además un papel activante básico para el polvo de vidrio.

\subsection{Ensayos realizados con cemento Portland}

Se fabrican una serie de probetas normalizadas (4) de mortero con conglomerante compuesto de polvo de vidrio y cemento Portland CEM I 52,5 R con distintas dosificaciones. Puesto que los ensayos de probetas fabricadas con conglomerante compuesto tan solo por sosa o potasa, polvo de vidrio y cal, han puesto de manifiesto las bajas resistencias que se obtienen transcurrido un breve periodo de tiempo de fraguado para un polvo de vidrio de $d_{50}=33 \mu \mathrm{m}$, se reemplaza este por otro cuya granulometría es $\mathrm{d}_{50}=16 \mu \mathrm{m}$. Al igual que en el primer grupo de probetas en este, solamente se varía la naturaleza del conglomerante en los siguientes aspectos: 
- Granulometría del polvo de vidrio usado en como conglomerante, se sustituye el polvo de vidrio $\mathrm{d}_{50}=33 \mu \mathrm{m}$ por polvo de vidrio $d_{50}=16 \mu \mathrm{m}$. Se usa, por tanto, polvo de vidrio de granulometrías $\mathrm{d} 50=11 \mu \mathrm{m}$ y de d50 $=16 \mu \mathrm{m}$.

- El añadido o no de cal viva al cemento.

- La proporción de polvo de vidrio en el conglomerante con relación a la de cemento. Esta variará entre un 70 y un $90 \%$

La Tabla 4 muestra las diferentes mezclas de reactivos usados y las variaciones de resistencia a la compresión simple que se obtienen. Donde:

$\mathrm{Ca}=$ Cal viva

Cem = CEM I 52,5 R

$\mathrm{V}=$ Polvo de vidrio.
En la Figura 2 se ha representado la variación de la resistencia a compresión en el tiempo para las diferentes mezclas reactivas.

Como en el caso de la serie de probetas anteriores, en el que se añadía cal y potasa, en este caso la adición de cemento al conglomerante provoca un gran incremento de las resistencias mecánicas a corto plazo.

Al igual que para la anterior serie de probetas, en este caso se consiguen mayores resistencia para probetas fabricadas con conglomerante a base de polvo de vidrio de granulometría pequeña. Es decir, se obtienen mayores resistencias para polvo de vidrio de $\mathrm{d}_{50}=11 \mu \mathrm{m}$ que para polvo de vidrio de $\mathrm{d}_{50}=16 \mu \mathrm{m}$. Aunque esta diferencia es inferior que en el caso de morteros fabricados a base de cal y potasa y con polvo de vidrio de granulometría $d_{50}=33 \mu \mathrm{m}$.

Tabla 4. Resultados de las resistencias a la compresión simple de probetas de morteros normalizados en función de las mezclas reactivas utilizadas.

\begin{tabular}{|c|c|c|c|c|c|c|}
\hline \multirow{2}{*}{$\begin{array}{c}\begin{array}{c}\text { Tipo de } \\
\text { conglomerante }\end{array} \\
\begin{array}{c}\text { Tipo de } \\
\text { mezcla reactiva }\end{array}\end{array}$} & \multicolumn{2}{|c|}{$\begin{array}{l}\text { Conglomerante a base de } \\
\text { polvo de vidrio de } d_{50}=11 \mu \mathrm{m}\end{array}$} & \multicolumn{4}{|c|}{$\begin{array}{l}\text { Conglomerante a base de } \\
\text { polvo de vidrio de } d_{50}=16 \mu \mathrm{m}\end{array}$} \\
\hline & $\begin{array}{l}80 \mathrm{~V}-20 \mathrm{Cem} \\
11 \mu \mathrm{m}\end{array}$ & $\begin{array}{l}\text { 80V-10Cem } \\
-10 \mathrm{Ca} 11 \mu \mathrm{m}\end{array}$ & $\begin{array}{l}\text { 70V-30Cem } \\
16 \mu \mathrm{m}\end{array}$ & $\begin{array}{l}80 \mathrm{~V}-20 \mathrm{Cem} \\
16 \mu \mathrm{m}\end{array}$ & $\begin{array}{l}\text { 90V-10Cem } \\
16 \mu \mathrm{m}\end{array}$ & $\begin{array}{l}95 \mathrm{~V}-5 \mathrm{Cem} \\
16 \mu \mathrm{m}\end{array}$ \\
\hline$\%$ vidrio & 80 & 80 & 90 & 80 & 80 & 90 \\
\hline$\% \mathrm{cal}$ & 20 & 10 & 30 & 20 & 10 & 5 \\
\hline$\%$ potasa & 0 & 10 & 0 & 0 & 2 & 0 \\
\hline $\mathrm{RC}$ (28 días) en MPa & 3,6 & 2,2 & 5 & 3,2 & 1,6 & 1,1 \\
\hline $\mathrm{RC}$ (90 días) en $\mathrm{MPa}$ & 15,1 & 9,8 & 15,9 & 11,6 & 6,5 & 3,5 \\
\hline RC (180 días) en MPa & 26,3 & 22,3 & 29,6 & 22 & 11,4 & 6,9 \\
\hline $\mathrm{RC}$ (360 días) en MPa & 30,0 & 25,6 & 34,8 & 25,6 & 13,3 & 8,1 \\
\hline
\end{tabular}

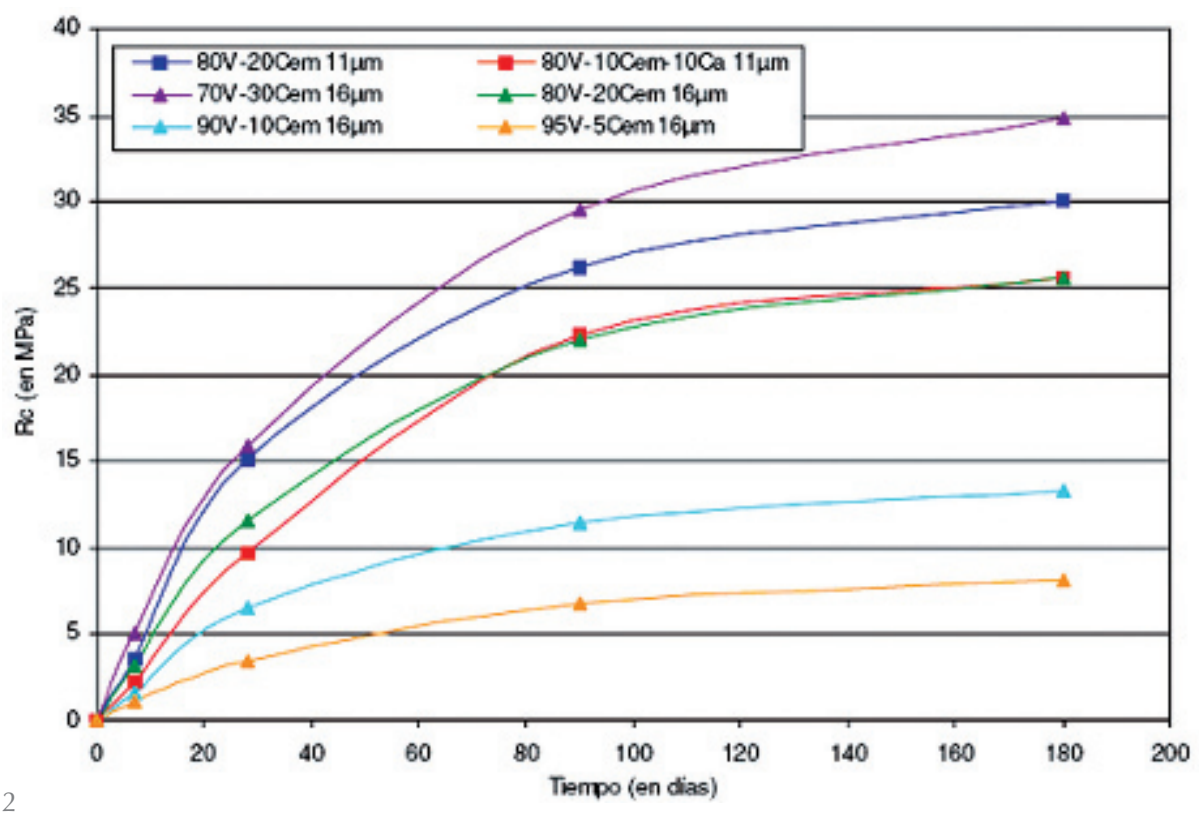


3. Variación de la resistencia a compresión de morteros en función del \% de polvo de vidrio en el conglomerante.
Nota: para el polvo de vidrio de d50 $=11 \mu \mathrm{m}$, la curva es una recta porque sólo se han tomado dos puntos de medida.
Por tanto, vuelve a quedar patente la influencia de la granulometría del polvo de vidrio en el comportamiento mecánico de los morteros.

Se observa en este caso, al igual que para los morteros a base de cal y potasa, que una dosificación mayor en reactivo permite alcanzar mayores resistencias a compresión. De esta forma, al sustituir un $10 \%$ de cemento por un $10 \%$ de cal viva en el conglomerante, se origina una disminución de la resistencia de entre un 15 y un 35\% dependiendo del plazo de tiempo transcurrido.

Al igual que para el anterior grupo de probetas ensayadas, cuyo conglomerante estaba formado por polvo de vidrio cal y potasa, en este segundo grupo de probetas ensayadas fabricadas con cemento CEM I 52,5 R (5), se aprecia un aumento de las resistencias a largo plazo, ya que esta continúa incrementándose aún entre los 180 y 360 días.

En este caso se observa que la granulomeya que el aumento de las resistencias a compresión se sigue incrementando en el tiempo independientemente de la granulometría del vidrio empleado y de la cantidad de polvo de vidrio contenido en el conglomerante. El crecimiento parece ser proporcional al tiempo, salvo al principio de los ensayos para morteros fabricados con polvo de vidrio de granulometría $\mathrm{d}_{50}=11 \mu \mathrm{m}$ cuyos valores de resistencia aumentan muy rápidamente en los primeros días $\left(\mathrm{Rc}_{28 \text { días }}\right.$ / $\mathrm{RC}_{7 \text { días }} \approx 4,3$ ), mientras que $\mathrm{Rc}_{90 \text { días }} / \mathrm{RC}_{28 \text { días }}$ $\approx 2$ y $\mathrm{RC}_{180 \text { días }} / \mathrm{RC}_{90 \text { días }} \approx 1,16$, para las diferentes mezclas reactivas.

Las probetas cuyo conglomerante se compone principalmente de polvo de vidrio altría del vidrio no tiene ninguna influencia,

canzan a los 28 días menos del 50\% de su resistencia a compresión final, sin embargo, las probetas cuyo conglomerante se compone únicamente de cemento Pórtland CEM I $52,5 \mathrm{R}$ alcanzan el $90 \%$ de su resistencia final en el mismo periodo de tiempo. Este comportamiento es también independiente de la granulometría del polvo de vidrio y de su proporción en el conglomerante, lo que de nuevo pone de manifiesto la alta reactividad del polvo de vidrio a largo plazo. En la Figura 3 se muestran las resistencias a compresión obtenidas con las diferentes mezclas reactivas, dependiendo del porcentaje de vidrio y de cemento contenido en el conglomerante.

$\mathrm{Al}$ igual que en el caso anterior, de las curvas de variación de la resistencia a compresión con el tiempo, se observa que el aumento del valor de la resistencia va ligando al aumento de la proporción de cemento con relación al polvo de vidrio contenido en el conglomerante. Se observa que el crecimiento de las resistencias a compresión obedece a una ley casi lineal para porcentajes de vidrio comprendidos entre el 70 y el $100 \%$. Este comportamiento es más fidedigno cuanto más corto es el periodo de tiempo transcurrido, tal y como se muestra en la Tabla 5.

Tabla 5. Previsión de las resistencias a compresión de los morteros en los que el conglomerante está compuesto de más de $70 \%$ de polvo de vidrio de $d_{50}=16 \mu \mathrm{m}$ (a 7, 28, 90 y180 días) (V designa el porcentaje de polvo de vidrio en el conglomerante).

\begin{tabular}{|c|c|}
\hline PLAZOS & ECUACIONES \\
\hline 7 días & $\mathrm{Rc}=-2,3.10-3 \times \mathrm{V}^{2}+0,22 \times \mathrm{V}$ \\
\hline 28 días & $\mathrm{Rc}=-7,5.10-3 \times \mathrm{V}^{2}+0,74 \times \mathrm{V}$ \\
\hline 90 días & $\mathrm{Rc}=-1,4.10-2 \times \mathrm{V}^{2}+1,39 \times \mathrm{V}$ \\
\hline 180 días & $\mathrm{Rc}=-1,64.10-2 \times \mathrm{V}^{2}+1,63 \times \mathrm{V}$ \\
\hline
\end{tabular}

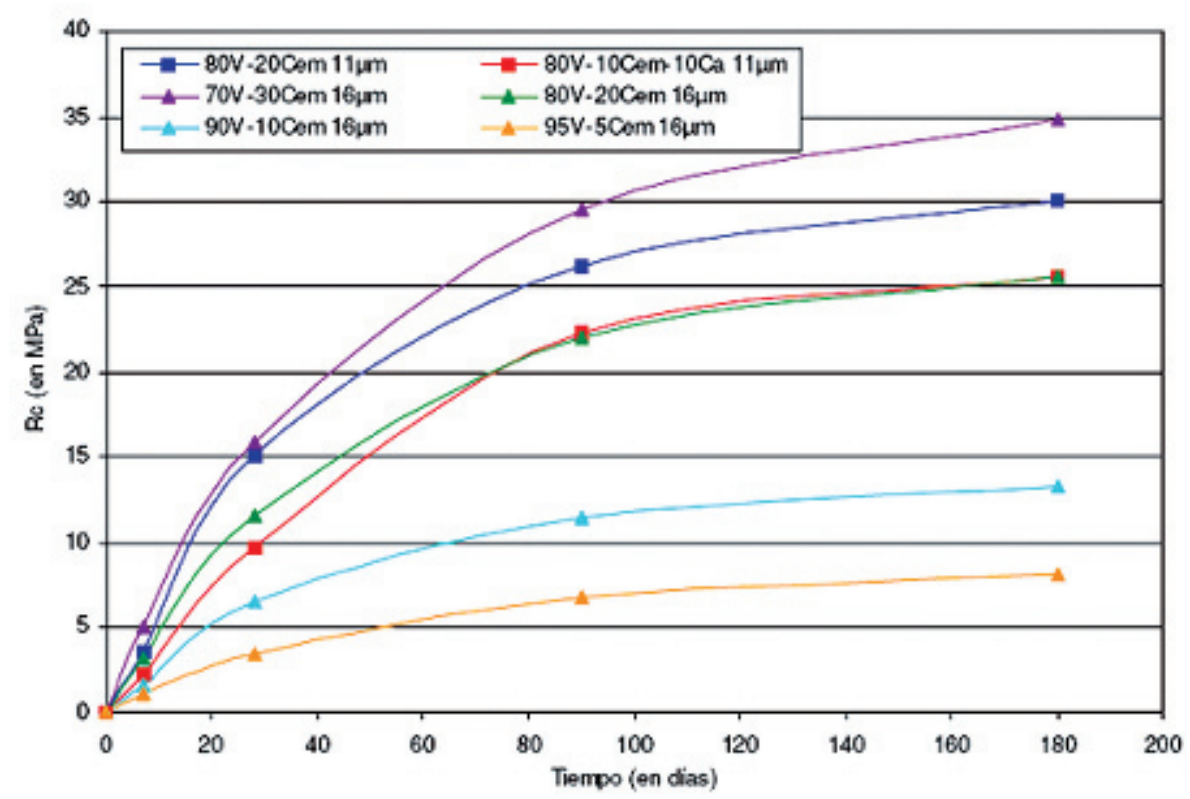


Finalmente resaltar, como se muestra en la Figura 3, el importante crecimiento de las resistencias a compresión de los morteros hasta los 180 días, independientemente del porcentaje de polvo de vidrio que contenga el conglomerante.

En la Figura 4 se ha representado la evolución de las resistencias a compresión para los morteros en los que el conglomerante se constituye por polvo de vidrio, CEM $52,5 \mathrm{R}$ y cal viva en las siguientes proporciones: $80 \%$ polvo de vidrio $20 \%$ de cal viva y CEM I 52,5 R. Este gráfico destaca el comportamiento del cemento CEM I 52,5 $\mathrm{R}$ en relación con el de la cal viva en las muestras reactivas analizadas.

Los morteros fabricados con cemento CEM I 52,5 R presentan, con el tiempo, resistencias a compresión de 1,5 a 6 veces mayores que las de los morteros a base de cal viva. Con una diferencia constante de aproximadamente, 12 MPa entre los 28 y los 180 días.

Entre estas dos clases de mortero (los fabricados con cal y los fabricados con cemento que constituyen el conglomerante), quedan patentes diferencias cinéticas de reacción. Mientras que para los morteros a base de cemento el aumento más rápido de las resistencias a compresión se produce entre los 0 y los 28 días, para los morteros constituidos a base de cal viva, la mayor velocidad de crecimiento de la resistencia a compresión se producirá entre los 28 y los 90 días.

Para verificar el papel que el polvo de vidrio desempeña en el crecimiento de las resistencias mecánicas, se fabricaron unas probetas en las que el conglomerante está formado por un $80 \%$ de polvo silíceo de granulometría $\mathrm{d}_{50}=24 \mu \mathrm{m}$, inerte, sustituyendo al polvo de vidrio y de un 20\% de cemento Portland CEM I 52,5 R.
En este caso las resistencias obtenidas son muy bajas en relación a las obtenidas para probetas fabricadas con las mismas proporciones pero sustituyendo el polvo fino silíceo por polvo de vidrio: $\mathrm{Rc}_{28 \text { días }}=2,8 \mathrm{MPa}$ y $\mathrm{RC}_{90 \text { ins }}=3,2 \mathrm{MPa}$ frente a 11,6 MPa y $22 \mathrm{MPa}$, obtenidas para las probetas en las que el conglomerante contiene el polvo de vidrio de $\mathrm{d}_{50}=16 \mu \mathrm{m}$.

Por lo tanto queda de manifiesto, una vez más, la importancia del polvo de vidrio en el aumento de las resistencias mecánicas de los morteros.

Para comprobar que el polvo fino silíceo utilizado no interviene en medida alguna en el fenómeno del fraguado, se prepararon unas probetas de mortero sustituyendo dicho material por otro de las similares características granulométricas pero de naturaleza caliza. En la Tabla 6 se reflejan los resultados obtenidos en los ensayos realizados con ambas mezclas.

En la Tabla 6 queda patente que las resistencias para los morteros elaborados a partir de los dos materiales son muy similares, por lo que, se descarta la hipótesis de la influencia de la arena del mortero como agente responsable del fenómeno del fraguado.

Tabla 6. Comparación de la evolución de las resistencias a compresión entre morteros constituidos de arena caliza y morteros compuestos de arena normalizada silícea en función del tiempo (conglomerante con $80 \%$ de PCLM de d50 = $16 \mu \mathrm{m}$ y $20 \%$ de cemento CEM I 52,5 R)

\begin{tabular}{|c|c|c|}
\hline Resistencia a compresión & Arena Caliza & Arena normalizada silícea \\
\hline Rc (7 días) en $\mathrm{MPa}$ & 3,4 & 3,2 \\
\hline Rc (7 días) en $\mathrm{MPa}$ & 13,8 & 11,6 \\
\hline Rc (7 días) en $\mathrm{MPa}$ & 22,4 & 22,0 \\
\hline Rc (7 días) en $\mathrm{MPa}$ & 25,7 & 25,6 \\
\hline
\end{tabular}

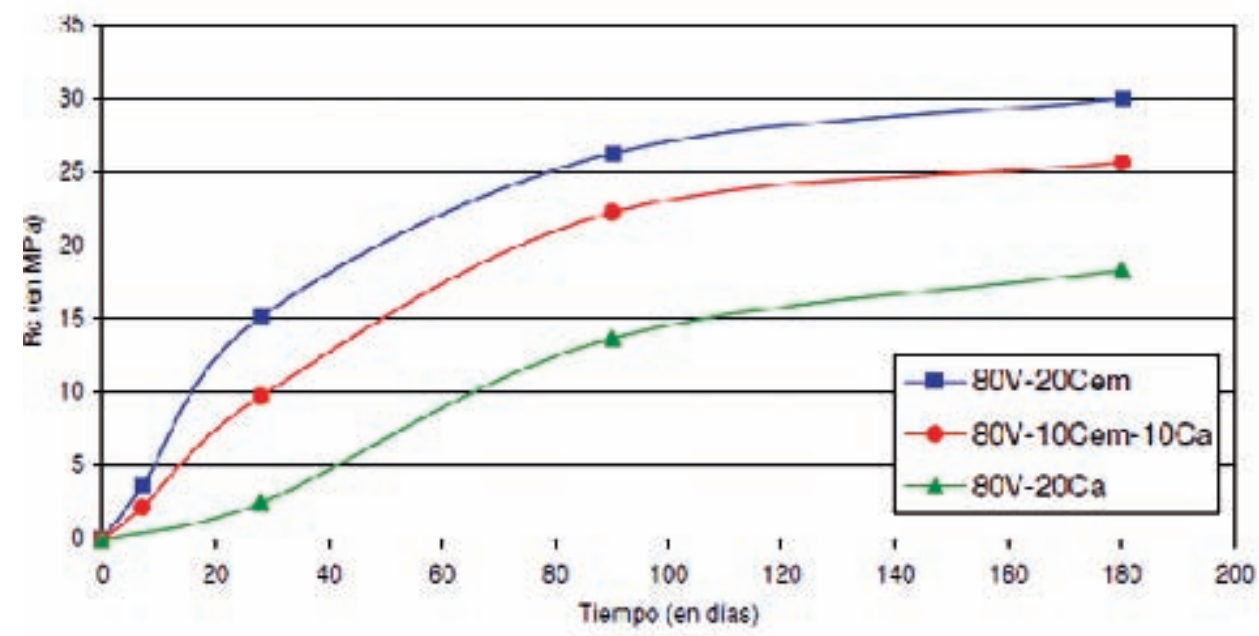




\section{CONCLUSIONES}

- La obtención de altos valores de resistencia a compresión simple de los morteros en los que el conglomerante está compuesto fundamentalmente de polvo de vidrio, indica que el vidrio molido interviene en su crecimiento.

- A lo largo de este estudio queda de manifiesto que las resistencias serán tanto más altas cuanto más pequeña sea la granulometría del polvo de vidrio empleado como conglomerante, y mayor sea la cantidad de este.

- Además del vidrio, el empleo de cemento Portland CEM I 52,5 R como reactivo, permite obtener resistencias mucho más altas que las obtenidas utilizando cal viva.

- A pesar de todo, el empleo de cal viva incluyendo una pequeña cantidad de potasa, es muy interesante cuando lo que se busca es obtener una evolución de las resistencias mecánicas a muy largo plazo, como es el caso de la estabilización de suelos.

- Por las especiales características expuestas a lo largo de este trabajo se puede concluir que, los morteros fabricados a partir de polvo de vidrio, cemento y cal, en las proporciones adecuadas, constituyen un material idóneo para ser usado como conglomerante en la estabilización de suelos. Destacando la prolongación en el tiempo del proceso de fraguado, que minimiza el riesgo de aparición de grietas en el pavimento.

- En este mismo campo, el de la estabilización de suelos, destacan sus propiedades ecológicas, ya que minimiza los impactos paisajísticos que causaría el empleo de conglomerantes tradicionales. El empleo del polvo de vidrio como conglomerante provoca que el color de los caminos estabilizados con este tipo de material, se integre perfectamente en el paisaje, ya que adquiere el color del árido empleado.

- Se trata por tanto de un material altamente ecológico, cuyo uso hace que se minimice el empleo de vertederos para el almacenamiento de los desechos de vidrio que la industria vidriera no puede procesar para su reciclaje, y además hace que disminuya el impacto paisajístico que lleva asociada la construcción de cualquier camino o vía de comunicación.

\section{BIBLIOGRAFÍA}

(1) Ahmad, S.; Aimin, X.: "Value-added utilisation of waste glass in concrete". Cement and Concrete Research. Elsevier (2003). Cement and Concrete Research, Vol. $34 \mathrm{n}^{\circ} 1$ (2004), pp. 81-89.

(2) Bashart, T.; Ghassan, N.: "Properties of concrete contains mixed colour waste recycled glass as sand and cement replacement". Construction and Building Material. Elsevier. (2007). Construction and Building Materials, Vol. 22 nº 5 (2008), pp. 713-720.

(3) Herrick, J. E.; Jones, T. L.: "A dynamic cone penetrometer for measuring soil penetration resistance" Soil Sci. Soc. Am. Vol. 66 no 4 (2002), pp. 1320-1324.

(4) UNE 83-304-84: Ensayos de Hormigón. Rotura por compresión.

(5) Kjellsen, K. O.; Detwiller, R. J.: "Reaction Kinetics of Portland cement mortars hydrated at different temperatures". Cement and Concrete Research, Vol. 22 n 1 (1992), pp. 112-120.

(6) Seung, B. P.; Bong, C. L.; Jeong, H. K.: "Studies on mechanical properties of concrete containing waste aggregate" Construction and Building Material. Elsevier. (2004). Cement and Concrete Research, Vol. 34 n 12 (2004), pp. 2181-2189. 